\title{
Monocyte Expression Levels of Cytokines in Patients With Fibromyalgia Syndrome
}

\author{
Evrim ÖĞÜT, ${ }^{1}$ Ali GÜR, ${ }^{2}$ Burhan Fatih KOÇYİĞİT, ${ }^{2}$ Serdar ÖZTUZCU, ${ }^{3}$ Beyhan CENGİZ, \\ Özlem ALTINDAĞ, ${ }^{2}$ Hilal KARAGÜLLÜ, ${ }^{5}$ Savaş GÜRSOY ${ }^{2}$ \\ ${ }^{1}$ Department of Physical Medicine and Rehabilitation, Erenköy Physical Medicine and Rehabilitation Hospital, Istanbul, Turkey \\ ${ }^{2}$ Department of Physical Medicine and Rehabilitation, Medical Faculty of Gaziantep University, Gaziantep, Turkey \\ ${ }^{3}$ Department of Medical Biology, Medical Faculty of Gaziantep University, Gaziantep, Turkey \\ ${ }^{4}$ Department of Medical Physiology, Medical Faculty of Gaziantep University, Gaziantep, Turkey \\ ${ }^{5}$ Department of Physical Medicine and Rehabilitation, Muş State Hospital, Muş, Turkey
}

\begin{abstract}
Objectives: This study aims to determine monocyte expression levels of interleukin 1 beta (IL-1 $\beta$ ), IL-6, IL-8, and tumor necrosis factor alpha in fibromyalgia syndrome (FMS) patients and healthy females.

Patients and methods: A total of 80 female FMS patients and 50 healthy females were evaluated. Twelve of the FMS patients who did not meet the 1990 American College of Rheumatology criteria and 10 healthy controls were excluded. After exclusion 68 participants as FMS patient (mean age $41.41 \pm 7.14$ years; range 18 to 55 years) and 40 participants as control group (mean age $39.12 \pm 10.9$ years; range 18 to 55 years) were included in the study. For both groups, a detailed form was filled out which included information on age, body mass index, marital status, educational status, visual analog scale, fibromyalgia impact questionnaire, Hamilton anxiety scale, Hamilton depression scale, modified fatigue impact scale, and Nottingham health profile. Whole blood peripheral blood monocyte cells were drawn in ethylenediaminetetraacetic acid tubes from patient and control groups between 08:30 to 9:30 hours. Tumor necrosis factor alpha, IL-6, IL-8, and IL-1 $\beta$ expressions were evaluated using fluorescence-activated cell sorting flow cytometry device.

Results: No statistically significant difference was detected between the two groups in terms of average age, body mass index, and other demographic data ( $p>0.05$ ). Visual analog scale, Hamilton anxiety scale, Hamilton Depression Scale, fibromyalgia impact questionnaire, modified fatigue impact scale total score and Nottingham health profile total-subscore values were statistically significantly higher in the patient group than the control group $(p<0.001)$. No significant difference was found in expression of cytokines by monocytes in patients with FMS ( $p>0.05$ ).

Conclusion: Although FMS is not traditionally considered an inflammatory disorder, evidence for elevated inflammatory processes has been noted in some studies. Our results do not support any role of the inflammatory cytokines in the pathogenesis of FMS.

Keywords: Fibromyalgia; interleukin 1beta; interleukin 6; interleukin 8; tumor necrosis factor alpha.
\end{abstract}

Fibromyalgia syndrome (FMS) is a multisystemic disease characterized by a chronic widespread musculoskeletal pain. ${ }^{1}$ Chronic pain may be accompanied by sleep disorder, fatigue, morning stiffness, depression, anxiety, and autonomic disorders such as spastic colon and bladder disorders. ${ }^{2}$ FMS affects 3 to $4 \%$ of the society, 80 to $90 \%$ of the patients are female, and is generally observed between the ages of
30 to $50 .{ }^{3}$ FMS restricts social activities; causes excessive use of health sources, and loss of labor and production. ${ }^{4}$ The reason for widespread pain and pain sensitivity in patients with FMS has not been explained yet. Although it is suggested that peripheral and central mechanisms as well as genetic and environmental factors play role in etiopathogenesis, the mechanism has not been fully understood. ${ }^{5}$ There are no laboratory

Received: October 22, 2014 Accepted: May 09, 2015 Published online: August 10, 2015

Correspondence: Burhan Fatih Koçyiğit, MD. Gaziantep Üniversitesi Tıp Fakültesi Hastanesi Fiziksel Tıp ve Rehabilitasyon Anabilim Dalı, 27310 Şehitkamil, Gaziantep, Turkey. Tel: +90 505 - 9068638 e-mail: bfk2701@hotmail.com

(2015 Turkish League Against Rheumatism. All rights reserved. 
tests and/or radiographic tests used in FMS diagnosis. Although FMS is not an inflammatory process, with the assertion of its relationship with symptoms and signs of the syndrome, interest in cytokines has increased. One of the theories adopted in FMS etiopathogenesis is that cytokines play a role in both etiology and modulation of core symptoms. ${ }^{6}$ Core symptoms are pain, fatigue, and mental and sleep disorders. ${ }^{7,8}$ Cytokines such as interleukin 1 beta (IL-1 $\beta$ ), IL-6, IL-8, and tumor necrosis factor alpha (TNF- $\alpha$ ) have been reported to be directly effective in development of central and peripheral neuropathic pain.9-11 These cytokines contribute to psychological, behavioral and hormonal changes in the central nerve system which lead to and augment pain. These changes are frequently referred to as "disease response" and are similar to symptoms of patients with FMS. ${ }^{12}$

Some dissimilar results have been obtained in studies aiming to investigate the relationship of cytokines with FMS symptoms and their role in etiopathogenesis. ${ }^{10,13}$ We relate these conflicts to different measurement methods of cytokines and whether the cytokines were measured directly or indirectly. Thus, in this study, we aimed to determine monocyte expression levels of IL-1 $\beta$, IL-6, IL-8, and TNF- $\alpha$ in FMS patients and healthy females.

\section{PATIENTS AND METHODS}

A total of 80 female FMS patients, who applied to Gaziantep University Faculty of Medicine Sahinbey Research and Application Hospital Department of Physical Medicine and Rehabilitation Polyclinic between March 2010 and April 2011, and 50 healthy females, who did not have any systemic diseases and showed similarities with the patient group in terms of age and demographic characteristics, were included in the study. Twelve of the FMS patients who did not meet the 1990 American College of Rheumatology criteria and 10 healthy controls were excluded. After exclusion 68 participants as FMS patient (mean age $41.41 \pm 7.14$ years; range 18 to 55 years) and 40 participants as control group (mean age $39.12 \pm 10.9$ years; range 18 to 55 years) were included in the study.
Exclusion criteria included psychiatric disease history such as schizophrenia, paranoia, somatoform disorder etc., immune deficiency, malignity, diabetes mellitus, hypo/hyperthyroid, chronic infection and inflammation (tuberculosis, rheumatoid arthritis, systemic lupus erythematous, ulcerative colitis, Crohn disease, ankylosing spondylitis etc.), cardiac and renal disease, pregnant and lactating females, having had a major trauma and surgical operation and taken serotonin reuptake inhibitors such as fluoxetine in the last six weeks, agents such as monoamine oxidase inhibitors activating central nerve system in the last two weeks, and non-steroid antiinflammatory drugs and acetaminophen etc. in the last week. The study was executed thanks to the support of the Scientific Research Fund following the approval of the Ethics Committee of Gaziantep University, Faculty of Medicine (project no: TF.10.29) and informed consent of all participants were obtained.

Each member from both groups filled in and signed a detailed survey form that queries age, marital status, height, weight, body mass index (BMI), educational status, $10 \mathrm{~cm}$ visual analog scale, patient's and physician's global assessment of disease severity, fibromyalgia impact questionnaire (FIQ), Hamilton anxiety and depression assessment scale (HAM-A and HAM$\mathrm{D}$, respectively), modified fatigue impact scale (MFIS), and Nottingham health profile scale. Members of both groups were surveyed about morning stiffness at joints, chronic pain, local pain, reflected pain, subjective swelling history at joints, non-refreshing sleep, paresthesia, fatigue, female urethral syndrome, Raynaud phenomenon, distress, headache, dysmenorrhea, irritable bowel syndrome, depression, allergic rhinitis, and sicca complex.

Fibromyalgia impact questionnaire measures 10 different factors namely physical function, feeling well, not being able to go to work, having challenges at work, pain, fatigue, morning fatigue, stiffness, anxiety and depression. Except for feeling well, low scores indicate improvement and being less affected by disease. The possible maximum score for every subheading is 10 . Thus, the total maximum score is $100 .{ }^{14}$

Modified fatigue impact scale evaluates fatigue by 21 questions. It consists of three subsections. 
Each question is scored between 0 to 4 points. Total score is between 0 to 84 . The highest score gives the most severe fatigue value. ${ }^{15}$

Visual analog scale is used for the assessment of pain severity. The scale is $10 \mathrm{~cm}$ long and the patient marks the point $(0=$ no pain, $10=$ the most severe pain) on a vertical or horizontal line corresponding to severity of the pain.

Patient's and physician's global assessment of disease severity require the patient and physician to evaluate the disease severity. It progresses from 0 to 10 . Zero means no pain and 10 means very severe pain.

Nottingham Health Profile evaluates health condition. Subheadings provide information about the activity of the patient. It consists of 38 questions regarding pain (eight questions), physical activity (eight questions), fatigue (three questions), sleep (five questions), social isolation (five questions), and emotional reactions (nine questions). Questions are answered as yes/no. Scores range between 0 (the best score) and 100 (the worst score). ${ }^{16}$

Hamilton Depression Scale assesses depression level of the patient. It consists of 17 questions and 14 points and over indicate depression. ${ }^{17}$ HAM-A has been arranged to determine the anxiety level and distribution of indications and to measure severity change. It consists of 14 items which question both mental and physical indications. Existence and severity of the items in the scale are evaluated by the interviewer. ${ }^{18}$

Analyses were performed on mononuclear cells in whole blood or peripheral blood samples taken from patient and control groups between 08:30 to 09:30 hours and placed into ethylenediaminetetraacetic acid tubes.

\section{Materials/kits used}

1. Brefeldin A was prepared with $5 \mathrm{mg} / \mathrm{mL}$ dimethyl sulfoxide (BD Biosciences, San Jose, CA, USA).

2. Lipopolysaccharide (LPS) was prepared with $5 \mathrm{mg} / \mathrm{mL}$ dimethyl sulfoxide/ phosphate buffered saline (PBS).

3. Monoclonal antibodies. CD14 antibody was used to express monocytes.
4. Fluorescence-activated cell sorting (FACS) lysing solution (10X) was diluted with 1:10 deionized water (BD Biosciences, San Jose, CA, USA).

5. Fluorescence-activated cell sorting permeabilizing solution (10X) was diluted with 1: 10 deionized water (BD Biosciences, San Jose, CA, USA).

6. Irrigation solution (Wash Buffer-PBS).

7. Fast immune anti-cytokine and control monoclonal antibodies (BD Biosciences, San Jose, CA, USA).

8. $1 \%$ paraformaldehyde.

Two different groups as unstimulated and activated cultures were prepared for monocyte activation. Unstimulated tubes were prepared by adding $500 \mu \mathrm{L}$ Roswell Park Memorial Institute (RPMI) solution and $2 \mu \mathrm{L}$ brefeldin $\mathrm{A}$ (BD Biosciences, San Jose, CA, USA) into $500 \mu \mathrm{L}$ whole blood with ethylenediaminetetraacetic acid. Activated tubes were prepared by adding $500 \mu \mathrm{L}$ RPMI solution and $2 \mu$ Lrefeldin A (BDBiosciences, San Jose, CA, USA) and $2 \mu \mathrm{L}$ LPS into $500 \mu \mathrm{L}$ whole blood with ethylenediaminetetraacetic acid. Tubes prepared for both groups were vortexed and incubated in 7\% carbon dioxide incubator at $37^{\circ} \mathrm{C}$ for four hours.

At the end of incubation, $50 \mu \mathrm{L}$ blood and $5 \mu \mathrm{L}$ CD14 antibody were included in Falcon tubes (Taizhou Runlab Labware Manufacturing Co., Ltd., Shaoxing, China) prepared for both groups and these were vortexed. They were incubated for 15-30 minutes at room temperature in the dark. At the end of incubation, $2 \mathrm{~mL} \mathrm{1X} \mathrm{FACS}$ Lysing Solution (BD Biosciences, San Jose, CA, USA) was included in the tubes, they were vortexed and incubated for 10 minutes at room temperature in the dark. Upon completion of incubation time, tubes were centrifuged at $1,500 \mathrm{rpm}$ for five minutes and the supernatant was removed. $500 \mu \mathrm{L}$ 1X FACS Permeabilizing solution (BD Biosciences, San Jose, CA, USA) was included on the pallet, it was vortexed and incubated for 10 minutes at room temperature in the dark. Two to $4 \mathrm{~mL}$ PBS was added to them, they were centrifuged at $1,500 \mathrm{rpm}$ for five minutes and the supernatant was removed. Five microliter $1 \%$ fluorescent conjugated monoclonal antibodies (TNF- $\alpha$, IL-6, IL-8, IL-1 $\beta$ ) (BD Biosciences, San Jose, CA, USA) were 
included on the pallets; they were centrifuged for 30 minutes at room temperature in the dark. At the end of incubation time, 2 to $4 \mathrm{~mL}$ PBS was added into Falcon tubes, they were centrifuged at $1,500 \mathrm{rpm}$ for five minutes and the supernatants were removed. $500 \mu \mathrm{L} 1 \%$ paraformaldehyde was added into the pellets. The prepared tubes were read by FACS Flow Cytometry (FACSCalibur, BD Biosciences, San Diego, CA, USA) and analyzed. One of the results is provided as an example in Figure 1.

\section{Statistical analysis}

Statistical analysis of data was performed using SPSS for Windows version 15.0 software program (SPSS Inc., Chicago, IL, USA). Chi square test was used to evaluate the difference between groups in terms of categorical variables. Data were normally distributed. Student t-test was used to compare mean values of the patient and the control groups. Correlations between different variables were analyzed by Pearson rank correlation. Statistical significance level was determined as 0.05 .
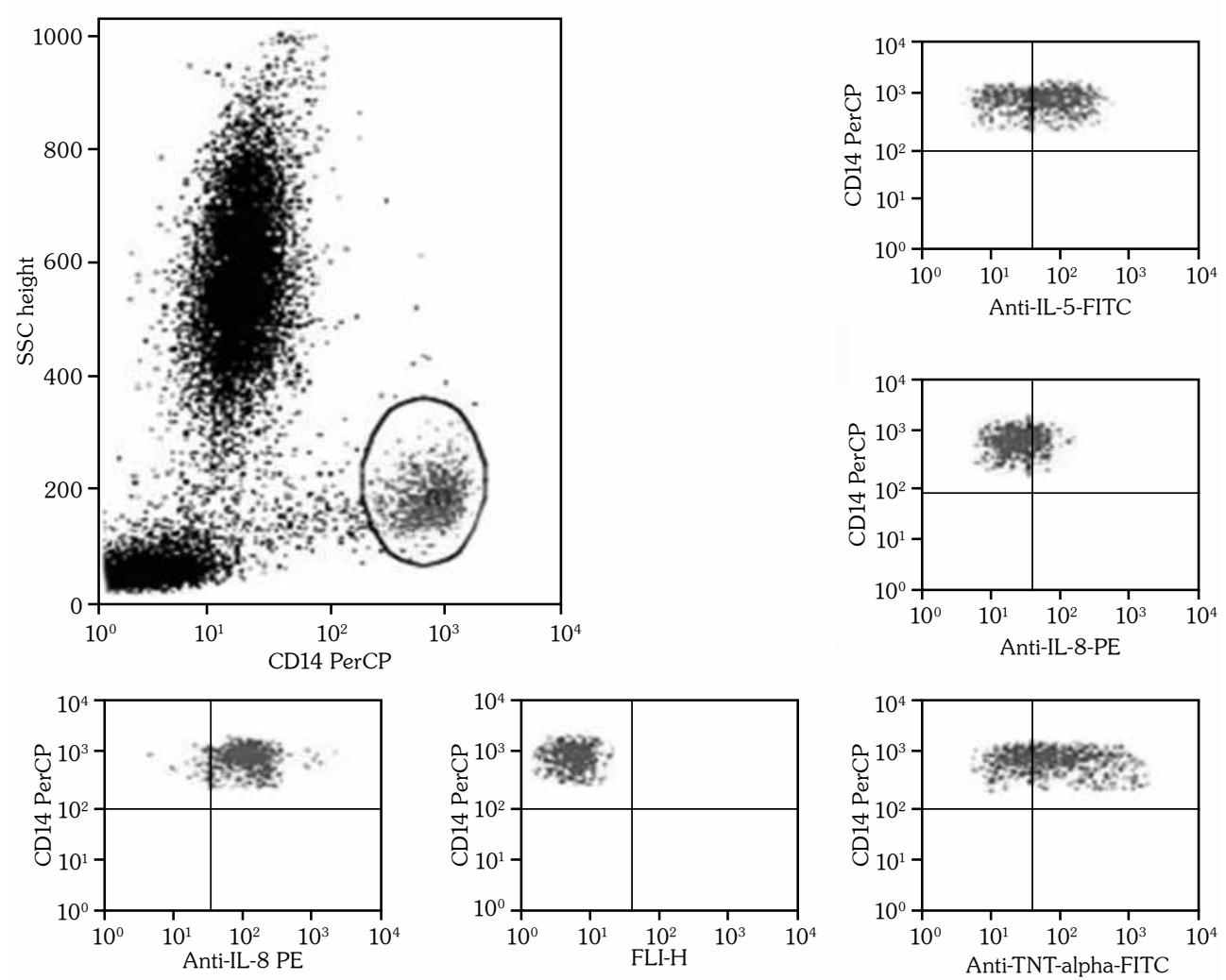

Figure 1. Sample results of a participant. PerCP: Peridinin chlorophyll; IL: Interleukin; TNF: Tumor necrosis factor.
No statistically significant difference was detected between the two groups in terms of average age, BMI, and other demographic data ( $p>0.05)$. BMIs of the patient and the control groups were $28.52 \pm 4.39$ and $26.92 \pm 6.38$, respectively which indicated no significant difference between the two groups ( $p>0.05)$. Demographic characteristics of the patient and the control groups are presented in Table 1. Prevalence of morning stiffness at joints, chronic pain, reflected pain, fatigue, non-refreshing sleep, subjective swelling history at joints, paresthesia, distress, headache, dysmenorrhea, irritable colon syndrome, and depression was higher and statistically significant in FMS patient group compared to the control group $(p<0.05)$ (Table 2).

Patient's and physician's global assessment of disease severity, visual analog scale, HAM-A, HAM-D, FIQ, MFIS total score, and Nottingham health profile-total subscore values were 


\begin{tabular}{|c|c|c|c|c|c|c|}
\hline & \multicolumn{3}{|c|}{ Patients $(n=68)$} & \multicolumn{3}{|c|}{ Controls $(n=40)$} \\
\hline & $\mathrm{n}$ & $\%$ & Mean & $\mathrm{n}$ & $\%$ & Mean \\
\hline Age (years) & & & 41.41 & & & 39.12 \\
\hline Body mass index $\left(\mathrm{kg} / \mathrm{m}^{2}\right)$ & & & 28.52 & & & 26.92 \\
\hline \multicolumn{7}{|l|}{ Educational status } \\
\hline Illiterate & 5 & 7.3 & & 0 & 0 & \\
\hline Literate & 6 & 8.8 & & 6 & 15 & \\
\hline Primary school graduate & 31 & 45.5 & & 9 & 22.5 & \\
\hline Secondary school graduate & 8 & 11.7 & & 5 & 12.5 & \\
\hline High school graduate & 10 & 14.7 & & 8 & 20 & \\
\hline University graduate & 6 & 8.8 & & 12 & 30 & \\
\hline Master's/doctor's degree & 2 & 2.9 & & 0 & 0 & \\
\hline \multicolumn{7}{|l|}{ Marital status } \\
\hline Married & 61 & 89.7 & & 31 & 77.5 & \\
\hline Single & 2 & 2.9 & & 8 & 20 & \\
\hline Widow & 2 & 2.9 & & 1 & 2.5 & \\
\hline Divorced & 2 & 2.9 & & 0 & 0 & \\
\hline Married/lives apart & 1 & 1.4 & & 0 & 0 & \\
\hline
\end{tabular}

significantly higher in the patient group than the control group $(p<0.001)$ (Table 3). There was no significant difference between the groups in terms of expression values of TNF- $\alpha$, IL-1 $\beta$, IL- 6 , and IL-8 ( $>0.05)$ (Table 4).

Significant positive correlations were shown between cytokines and Nottingham health profile subscales in FMS patients (Table 5). No significant correlations were found between cytokines and visual analog scale, patient's and physician's global assessment of disease activity, FIQ, HAM-A, HAM-D, and MFIS scores $(p>0.05)$.
Patients with HAM-A and HAM-D scores over 14, FIQ scores between 50 to 69, and FIQ scores over 69 were divided into subgroups. When these subgroups were compared with control group, there was no significant difference in terms of expressed amounts of cytokines ( $p>0.05)$.

\section{DISCUSSION}

Fibromyalgia syndrome is a chronic pain syndrome accompanied by widespread muscle pain, fatigue, and sleep disorder that last longer than three months. There have been

Table 2. Symptoms and findings in patient and control groups

\begin{tabular}{|c|c|c|c|c|c|}
\hline & \multicolumn{2}{|c|}{ Patients $(n=68)$} & \multicolumn{2}{|c|}{ Controls $(n=40)$} & \multirow[b]{2}{*}{$p$} \\
\hline & $\mathrm{n}$ & $\%$ & $\mathrm{n}$ & $\%$ & \\
\hline Morning stiffness in joints & 46 & 67.6 & 2 & 5 & $<0.001$ \\
\hline Chronic pain & 68 & 100 & 8 & 20 & $<0.001$ \\
\hline Local pain & 55 & 80.8 & 27 & 67.5 & 0.162 \\
\hline Reflected pain & 44 & 64.7 & 15 & 37.5 & 0.009 \\
\hline Subjective swelling history at joints & 50 & 73.5 & 3 & 7.5 & $<0.001$ \\
\hline Non-refreshing sleep & 64 & 94.1 & 7 & 17.5 & $<0.001$ \\
\hline Paresthesia & 55 & 80.8 & 8 & 20 & $<0.001$ \\
\hline Fatigue & 67 & 98.5 & 25 & 62.5 & $<0.001$ \\
\hline Woman urethral syndrome & 20 & 29.4 & 6 & 15 & 0.107 \\
\hline Raynaud's phenomenon & 0 & 0 & 1 & 2.5 & 0.37 \\
\hline Distress & 62 & 91.1 & 14 & 35 & $<0.001$ \\
\hline Headache & 51 & 75 & 10 & 25 & $<0.001$ \\
\hline Dysmenorrhea & 26 & 38.2 & 7 & 17.5 & 0.031 \\
\hline Irritable bowel syndrome & 42 & 61.7 & 14 & 35 & 0.01 \\
\hline Depression & 55 & 80.8 & 10 & 25 & $<0.001$ \\
\hline Allergic rhinitis & 6 & 8.8 & 2 & 5 & 0.707 \\
\hline Sicca complex & 11 & 16.1 & 2 & 5 & 0.126 \\
\hline
\end{tabular}


Table 3. Clinic characteristics of patient and control groups

\begin{tabular}{|c|c|c|c|}
\hline & \multirow{2}{*}{$\begin{array}{c}\text { Patients } \\
\text { Mean } \pm S D\end{array}$} & \multirow{2}{*}{$\begin{array}{c}\text { Controls } \\
\text { Mean } \pm \text { SD }\end{array}$} & \multirow[b]{2}{*}{$p$} \\
\hline & & & \\
\hline Patient's global assessment of disease activity, VAS $(\mathrm{cm})$ & $6.17 \pm 1.62$ & $2.00 \pm 1.56$ & $<0.001$ \\
\hline Physician's global assessment of disease activity, VAS $(\mathrm{cm})$ & $5.16 \pm 1.14$ & $1.00 \pm 0.96$ & $<0.001$ \\
\hline Pain severity, VAS $(\mathrm{cm})$ & $6.63 \pm 1.72$ & $2.52 \pm 1.50$ & $<0.001$ \\
\hline Fibromyalgia impact questionnaire-total score & $51.77 \pm 11.15$ & $19.32 \pm 9.44$ & $<0.001$ \\
\hline Hamilton anxiety assessment scale total score & $14.23 \pm 6.40$ & $8.02 \pm 3.77$ & $<0.001$ \\
\hline Hamilton depression assessment scale total score & $12.51 \pm 5.36$ & $6.55 \pm 3.44$ & $<0.001$ \\
\hline Modified fatigue impact scale-total score & $46.55 \pm 14.58$ & $20.35 \pm 12.49$ & $<0.001$ \\
\hline Nottingham health profile-pain & $59.20 \pm 26.38$ & $16.31 \pm 15.30$ & $<0.001$ \\
\hline Nottingham health profile-physical & $36.11 \pm 17.78$ & $12.98 \pm 11.10$ & $<0.001$ \\
\hline Nottingham health profile-fatigue & $80.94 \pm 27.37$ & $20.16 \pm 29.37$ & $<0.001$ \\
\hline Nottingham health profile-sleep & $50.92 \pm 26.60$ & $13.73 \pm 14.81$ & $<0.001$ \\
\hline Nottingham health profile-social & $31.40 \pm 31.40$ & $6.65 \pm 15.02$ & $<0.001$ \\
\hline Nottingham health profile-emotional & $44.83 \pm 28.53$ & $9.83 \pm 13.99$ & $<0.001$ \\
\hline
\end{tabular}

multi-lateral researches and discussions on FMS which needs a sophisticated and challenging treatment. One of the prominent theories concerning FMS is that cytokines play a role both in etiology and severity of core systems. Attention towards cytokines as the potential mediator of core symptoms in FMS was drawn when Wallace et al. ${ }^{19}$ reported acute onset of FMS symptoms including pain, fatigue, and mental state and sleep disorder in cancer patients receiving IL-2 treatment. Cytokines are immune-modulator proteins and have vast biologic effects. ${ }^{20}$ While IL-8 triggers sympathetic pain, IL- 6 induces hyperalgesia, fatigue, and depression. IL-1 $\beta$ affects hyperalgesia, fatigue, myalgia, and fever. Finally TNF- $\alpha$ is known to trigger rapid eye movement sleep, allodynia, and stress. ${ }^{21}$

In the literature, comparison of IL- $1 \beta$ levels in FMS and control groups showed no significant difference, which was also consistent with our study. ${ }^{6,10,22-25}$ Four of these studies measured serum levels of IL-1 $\beta,,^{10,22-24}$ while one study measured both serum and cytokine content of $\mathrm{PBMCs}^{6}$ and another conducted a cytokine expression measurement from PBMCs with a method similar to our FACS. ${ }^{25}$

Table 4. Comparison of tumor necrosis factor-alpha, interleukin 1- $\beta$, interleukin-6, and interleukin- 8 expression levels in monocytes of patient and the control groups

\begin{tabular}{|c|c|c|c|}
\hline & Patients & Controls & \\
\hline & Mean \pm SD & Mean \pm SD & $p$ \\
\hline Tumor necrosis factor-alpha & $367.04 \pm 130.72$ & $417.40 \pm 143.80$ & 0.065 \\
\hline Interleukin 1-beta & $459.97 \pm 149.80$ & $486.70 \pm 167.61$ & 0.394 \\
\hline Interleukin-6 & $313.32 \pm 128.98$ & $341.80 \pm 158.54$ & 0.312 \\
\hline Interleukin-8 & $259.67 \pm 165.56$ & $306.95 \pm 181.72$ & 0.170 \\
\hline
\end{tabular}

Table 5. Correlation analysis between cytokines and Nottingham health profile subscales

\begin{tabular}{|c|c|c|c|c|c|c|c|c|c|c|c|c|}
\hline & \multicolumn{2}{|c|}{ NHP-pain } & \multicolumn{2}{|c|}{ NHP-physical } & \multicolumn{2}{|c|}{ NHP-fatigue } & \multicolumn{2}{|c|}{ NHP-sleep } & \multicolumn{2}{|c|}{ NHP-social } & \multicolumn{2}{|c|}{ NHP-emotional } \\
\hline & $\mathrm{r}$ & $p$ & $r$ & $\mathrm{p}$ & $r$ & $\mathrm{p}$ & $\mathrm{r}$ & $\mathrm{p}$ & $\mathrm{r}$ & $\mathrm{p}$ & $\mathrm{r}$ & $\mathrm{p}$ \\
\hline TNF- $\alpha$ & -0.025 & 0.839 & -0.022 & 0.857 & 0.042 & 0.731 & $0.252^{*}$ & 0.038 & 0.205 & 0.094 & $0.239^{*}$ & 0.049 \\
\hline IL-1 $\beta$ & 0.78 & 0.530 & 0.82 & 0.506 & 0.189 & 0.123 & $0.267^{*}$ & 0.028 & $0.248^{*}$ & 0.042 & $0.317^{* * *}$ & 0.009 \\
\hline IL-6 & 0.024 & 0.846 & -0.002 & 0.985 & 0.068 & 0.581 & $0.272^{*}$ & 0.025 & 0.184 & 0.133 & 0.177 & 0.149 \\
\hline IL-8 & -0.030 & 0.806 & -0.031 & 0.802 & 0.014 & 0.913 & 0.177 & 0.149 & 0.069 & 0.576 & 0.099 & 0.422 \\
\hline
\end{tabular}

NHP: Nottingham health profile; TNF- $\alpha$ : Tumor necrosis factor-alpha; IL-1ß: Interleukin 1-beta; IL-6: Interleukin-6; IL-8: Interleukin-8. * Correlation is significant at the 0.05 level, ${ }^{* *}$ Correlation is significant at the 0.01 level. 
A study showed that IL-1 $\alpha$ levels of FMS patients were too low to be within the measurement range. ${ }^{26}$ There was no significant difference between 64 FMS patients and 25 healthy volunteers included in that study in terms of age, sex, ethnic origin, and other demographic data such as educational status and socioeconomic level. However, BMI in FMS patient group was significantly higher than the control group. This might be the reason why the results of that study are different from ours and other studies. Therefore, we tried to include individuals with similar demographical characteristics, i.e. age, sex, BMI, ethnic origin, and educational status, in both groups to prevent the results of our study from being affected by such factors.

In seven studies where IL-6 levels in FMS patients were examined, no significant differences were found between the patient and control groups. ${ }^{10,22,24,25,27-29}$ Five of these studies measured serum and plasma concentrations, ${ }^{10,22,27-29}$ one measured serum emission levels of IL-6 for 24 hours (while sleeping and awake), ${ }^{24}$ and another measured the levels expressed from PBMCs. ${ }^{25}$

Coherent with these studies, we did not obtain a significant difference between the patient and control groups in terms of IL- 6 expression from PBMCs at the end of our study. On the other hand, one study reported higher IL-6 levels in FMS patient group than the control group. ${ }^{6}$ The difference in this study was related to the applied method where comparison of cytokine generation from PBMCs was made depending on the response of the patient and the control group PBMCs to stimulation with in vitro lectin phytohemagglutinin (PHA) or LPS (with and without phorbol myristic acetate). Researchers demonstrated that the amount of IL- 6 produced in patient PBMCs stimulated with LPS was similar with the amount of IL- 6 in control group; while in presence of phorbol myristic acetate, the patient PBMCs stimulated with PHA acted differently from the control group.

Various mitogens were used to stimulate cytokine expression in different studies, including phorbol myristate acetate, concanavalin $\mathrm{A}$, and PHA. ${ }^{21}$ We used LPS in our study for this purpose. We demonstrated that cytokine expression from PBMCs stimulated with LPS did not show any difference between the patient and the control groups, which was similar to the results of the above-mentioned study. ${ }^{6}$ An analysis of the relationship between TNF- $\alpha$ and FMS revealed that the TNF- $\alpha$ level of the patient and the control groups were similar in five studies. , $23-25,30$ These studies compared the patient and control groups in terms of serum, ${ }^{23}$ serum and simultaneous PBMC generation, ${ }^{6} 24$ hour emission pattern in the plasma, ${ }^{24}$ serum and simultaneous messenger ribonucleic acid expressions, ${ }^{30}$ and intracellular cytokine levels. ${ }^{25}$ TNF- $\alpha$ levels expressed on the surface of PBMCs of the patient and the control groups were compared in our study and no significant difference was found.

Meanwhile, two studies discovered significantly higher TNF- $\alpha$ plasma and serum concentrations in FMS patients compared to the control group. ${ }^{27,28}$ Single measurement was made in one of these studies $^{27}$ whereas regular measurements were made throughout six months in the other. ${ }^{28}$

Contrary to these studies, there are studies which detected a lower TNF- $\alpha$ level in the patient group. ${ }^{26}$ Contradictory results of these studies which compared results from plasma and serum might be arising from the differences in patient selection criteria, number of participants, age, BMI, and measurement methods.

Furthermore, in four studies which examined the relationship between FMS and IL-8 level, 6,10,22,24,25,27-31 there was no significant difference between the measurements of the patient and the control groups. ${ }^{24,25,29,30}$ These studies measured emission levels of IL-8 in plasma for 24 hours, ${ }^{24}$ intracellular levels of PBMCs stimulated by PHA with the FACS method, ${ }^{25}$ with enzyme-linked immunosorbent assay in serum ${ }^{29}$ and both enzyme-linked immunosorbent assay in serum and messenger ribonucleic acid expressions simultaneously with polymerase chain reaction. ${ }^{30}$ We did not find any significant difference between the levels of IL-8 expressed on the surface of PBMCs in patient and control groups, either.

In other studies in this field, serum and plasma measurements were performed on samples taken only once and IL-8 levels were shown to be high in the patient group. ${ }^{10,22,27}$ In another study which found plasma IL-8 levels higher in the patients, no significant difference was detected in patient and control groups in terms of simultaneous in vitro PBMC production levels. ${ }^{6}$ In two different studies 
conducted by the same researcher group, serum IL-8 levels were measured in the samples taken on determined days during the six month monitoring period and were demonstrated to be significantly high in the patient group. ${ }^{28,31}$

Our study had some limitations. First, our sample size was small. We need further comprehensive studies with greater sample size where serum and PMBC related measurements are made together. Additionally, cytokine levels in the cerebrospinal fluid may provide more accurate information.

In many studies including ours where cytokine analyses were performed using methods such as PBMC related cell cytokine content, production levels, surface expression or messenger ribonucleic acid expression, no significant difference was found between the patient and control groups but only high levels of serum/plasma measuring were reported. This suggests that there is no difference in production and expressions from monocytes and there might be other factors affecting their serum/plasma cytokine levels and duration of existence and functions in peripheral structures.

The role of inflammation in fibromyalgia has been the topic of research and debate for decades. The overall literature regarding inflammatory processes in FMS is inconsistent and commonly includes conflicting results. Contradictory results obtained from studies that analyze the role of cytokines in FMS etiopathogenesis might be stemming from patient selection criteria, difference in number of participants, population characteristics, different symptom patterns, differences in measurement methods such as direct measurement or expression levels from serum/plasma or monocytes. Our study indicates that dysregulation of cytokines derived from circulating monocytes is not a dominant factor in the pathogenesis of FMS. Additionally, there was no significant difference in cytokine levels among patients with severe symptoms. However, the role of cytokines in the pathogenesis of FMS may not totally be excluded owing to the results obtained in either ex vivo or in vitro studies which do not necessarily represent the in vivo conditions. Also, symptoms in patients with FMS may be more related to disordered cytokine production by glial cells within the central nervous system than the production of cytokines in the circulation.

\section{Declaration of conflicting interests}

The authors declared no conflicts of interest with respect to the authorship and/or publication of this article.

\section{Funding}

The authors received no financial support for the research and/or authorship of this article.

\section{REFERENCES}

1. Rooks DS. Fibromyalgia treatment update. Curr Opin Rheumatol 2007;19:111-7.

2. Thompson ME, Barkhuizen A. Fibromyalgia, hepatitis $\mathrm{C}$ infection, and the cytokine connection. Curr Pain Headache Rep 2003;7:342-7.

3. Sarzi-Puttini P, Rizzi M, Andreoli A, Panni B, Pecis M, Colombo $\mathrm{S}$, et al. Hypersomnolence in fibromyalgia syndrome. Clin Exp Rheumatol 2002;20:69-72.

4. Knight T, Schaefer C, Chandran A, Zlateva G, Winkelmann A, Perrot S. Health-resource use and costs associated with fibromyalgia in France, Germany, and the United States. Clinicoecon Outcomes Res 2013;5:171-80.

5. Dadabhoy D, Clauw DJ. Therapy Insight: fibromyalgia--a different type of pain needing a different type of treatment. Nat Clin Pract Rheumatol 2006;2:364-72.

6. Wallace DJ, Linker-Israeli M, Hallegua D, Silverman S, Silver D, Weisman MH. Cytokines play an aetiopathogenetic role in fibromyalgia: a hypothesis and pilot study. Rheumatology (Oxford) 2001;40:743-9.

7. Mease P, Arnold LM, Bennett R, Boonen A, Buskila D, Carville S, et al. Mease P, Arnold LM, Bennett R, Boonen A, Buskila D, Carville S, et al. J Rheumatol 2007;34:1415-25.

8. Zautra AJ, Fasman R, Parish BP, Davis MC. Daily fatigue in women with osteoarthritis, rheumatoid arthritis, and fibromyalgia. Pain 2007;128:128-35.

9. Kwiatek R, Barnden L, Tedman R, Jarrett R, Chew J, Rowe C, et al. Regional cerebral blood flow in fibromyalgia: single-photon-emission computed tomography evidence of reduction in the pontine tegmentum and thalami. Arthritis Rheum 2000;43:2823-33.

10. Gür A, Karakoç M, Nas K, Remzi, Cevik, Denli $\mathrm{A}$, et al. Cytokines and depression in cases with fibromyalgia. J Rheumatol 2002;29:358-61.

11. Salemi S, Rethage J, Wollina U, Michel BA, Gay $\mathrm{RE}$, Gay S, et al. Detection of interleukin 1beta (IL-1beta), IL-6, and tumor necrosis factor-alpha in skin of patients with fibromyalgia. J Rheumatol 2003;30:146-50.

12. Kelley KW, Bluthé RM, Dantzer R, Zhou JH, Shen $\mathrm{WH}$, Johnson RW, et al. Cytokine-induced sickness behavior. Brain Behav Immun 2003;17:112-8. 
13. Uçeyler N, Häuser W, Sommer C. Systematic review with meta-analysis: cytokines in fibromyalgia syndrome. BMC Musculoskelet Disord 2011;12:245.

14. Sarmer S, Ergin S, Yavuzer G. The validity and reliability of the Turkish version of the Fibromyalgia Impact Questionnaire. Rheumatol Int 2000;20:9-12.

15. Noonan VK, Cook KF, Bamer AM, Choi SW, Kim $\mathrm{J}$, Amtmann D. Measuring fatigue in persons with multiple sclerosis: creating a crosswalk between the Modified Fatigue Impact Scale and the PROMIS Fatigue Short Form. Qual Life Res 2012;21:1123-33.

16. Kücükdeveci AA, McKenna SP, Kutlay S, Gürsel Y, Whalley D, Arasil T. The development and psychometric assessment of the Turkish version of the Nottingham Health Profile. Int $\mathrm{J}$ Rehabil Res 2000;23:31-8.

17. Akdemir A, Türkçapar MH, Orsel SD, Demirergi N, Dag I, Ozbay MH. Reliability and validity of the Turkish version of the Hamilton Depression Rating Scale. Compr Psychiatry 2001;42:161-5.

18. Hamilton M. The assessment of anxiety states by rating. $\mathrm{Br} \mathrm{J}$ Med Psychol 1959;32:50-5.

19. Wallace DJ, Margolin K, Waller P. Fibromyalgia and interleukin-2 therapy for malignancy. Ann Intern Med 1988;108:909.

20. Corwin EJ. Understanding cytokines. Part I: Physiology and mechanism of action. Biol Res Nurs 2000;2:30-40.

21. Menzies V, Lyon DE. Integrated review of the association of cytokines with fibromyalgia and fibromyalgia core symptoms. Biol Res Nurs 2010;11:387-94.

22. Gur A, Karakoc M, Erdogan S, Nas K, Cevik R, Sarac AJ. Regional cerebral blood flow and cytokines in young females with fibromyalgia. Clin Exp Rheumatol 2002;20:753-60.

23. Wallace DJ, Peter JB, Bowman RL, Wormsley $\mathrm{SB}$, Silverman S. Fibromyalgia, cytokines, fatigue syndromes, and immune regulation. In: Fricton JR, Awad E, editors. Advances in pain research and therapy. New York: Raven Pres; 1990. p. 277-87.

24. Togo F, Natelson BH, Adler GK, Ottenweller JE, Goldenberg DL, Struzik ZR, et al. Plasma cytokine fluctuations over time in healthy controls and patients with fibromyalgia. Exp Biol Med (Maywood) 2009;234:232-40.

25. Macedo JA, Hesse J, Turner JD, Ammerlaan W, Gierens A, Hellhammer DH, et al. Adhesion molecules and cytokine expression in fibromyalgia patients: increased L-selectin on monocytes and neutrophils. J Neuroimmunol 2007;188:159-66.

26. Hernandez ME, Becerril E, Perez M, Leff P, Anton B, Estrada S, et al. Proinflammatory cytokine levels in fibromyalgia patients are independent of body mass index. BMC Res Notes 2010;3:156.

27. Bazzichi L, Rossi A, Massimetti G, Giannaccini G, Giuliano T, De Feo F, et al. Cytokine patterns in fibromyalgia and their correlation with clinical manifestations. Clin Exp Rheumatol 2007;25:225-30.

28. Wang $H$, Moser $M$, Schiltenwolf $M$, Buchner $M$. Circulating cytokine levels compared to pain in patients with fibromyalgia -- a prospective longitudinal study over 6 months. J Rheumatol 2008;35:1366-70.

29. Xiao Y, Haynes WL, Michalek JE, Russell IJ. Elevated serum high-sensitivity C-reactive protein levels in fibromyalgia syndrome patients correlate with body mass index, interleukin- 6 , interleukin- 8 , erythrocyte sedimentation rate. Rheumatol Int 2013;33:1259-64.

30. Uçeyler N, Valenza R, Stock M, Schedel R, Sprotte G, Sommer C. Reduced levels of antiinflammatory cytokines in patients with chronic widespread pain. Arthritis Rheum 2006;54:2656-64.

31. Wang H, Buchner M, Moser MT, Daniel V, Schiltenwolf M. The role of IL- 8 in patients with fibromyalgia: a prospective longitudinal study of 6 months. Clin $\mathrm{J}$ Pain 2009;25:1-4. 